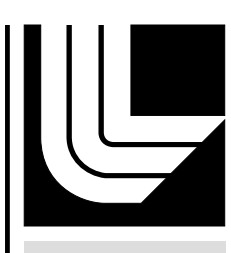

LAWRENCE LIVERM ORE N A TIO N A L LABORATORY

\title{
Shape-influenced Magnetic Properties of CoO Nanoparticles
}

S. Kundu, A. J. Nelson, S. K. McCall, A. W. VanBuuren, $\mathrm{H}$. Liang

March 8, 2013

Journal of Nanoparticle Research 
This document was prepared as an account of work sponsored by an agency of the United States government. Neither the United States government nor Lawrence Livermore National Security, LLC, nor any of their employees makes any warranty, expressed or implied, or assumes any legal liability or responsibility for the accuracy, completeness, or usefulness of any information, apparatus, product, or process disclosed, or represents that its use would not infringe privately owned rights. Reference herein to any specific commercial product, process, or service by trade name, trademark, manufacturer, or otherwise does not necessarily constitute or imply its endorsement, recommendation, or favoring by the United States government or Lawrence Livermore National Security, LLC. The views and opinions of authors expressed herein do not necessarily state or reflect those of the United States government or Lawrence Livermore National Security, LLC, and shall not be used for advertising or product endorsement purposes. 


\title{
Shape-influenced Magnetic Properties of CoO Nanoparticles
}

\author{
Subrata Kundu, ${ }^{+*}$ Art J. Nelson, ${ }^{\#}$ Scott McCall, \\ Anthony van Buuren, ${ }^{\#}$ and Hong Liang** \\ *Materials Science \& Mechanical Engineering, Texas A\&M University, College Station, TX \\ 77843-3123 (USA) \\ \#Lawrence Livermore National Laboratory, Physical \& Life Sciences Directorate, Condensed \\ Matter \& Materials Division, 7000 East Avenue, Livermore, CA 94550 (USA). \\ ${ }^{\dagger}$ Electrochemical Materials Science (ECMS) Division, CSIR-Central Electrochemical Research \\ Institute (CSIR-CECRI), Karaikudi-630006, Tamil Nadu, INDIA.
}

*Corresponding authors E-mails: skundu@cecri.res.in and hliang@tamu.edu,Phone-979-

862-2623, Fax - 979-845-3081

\begin{abstract}
Using a wet chemical approach, $\mathrm{CoO}$ nanospheres, nano-rings, nano-flowers, and nanowires of different sizes were generated. Among those, nano-rings show ferromagnetic behavior below $6 \mathrm{~K}$ while the nanospheres remain paramagnetic down. X-ray photoelectron spectroscopy for Co $2 \mathrm{p}, 3 \mathrm{p}$ and $3 \mathrm{~s}$ core-levels indicates the paramagnetic high-spin Co(II) electronic configuration. This finding reveals the optical, electronic, and magnetic behavior of $\mathrm{CoO}$ nanoparticles (NPs) that opens new opportunities for future applications as catalysts precursors for making pigments, lithium-ion battery materials, or as solid state sensors as anisotropy source for magnetic recording.
\end{abstract}

Keywords: Magnetic nanoparticles . CoO nanostructures . Paramagnetic . ferromagnetic . Electronic structures. 


\section{Introduction}

With the change in size and shape, nanoparticles exhibit significant changes in optical, electronic, catalytic, and magnetic properties (Alivisatos 1996, Kundu and Liang 2008, Kundu et al. 2009, Mandal et al. 2003). Shape-selective synthesis of nanomaterials, such as nanorods (Jana et al. 2001), nanowires (Chen et al. 2007), nanoprisms (Kundu et al. 2008), etc. shows tremendous promise for a wide variety of applications. In terms of synthesis, the wet chemical method has advantages over other physical methods in controlling the size and shape of the particles, in which the metal ions are reduced and stabilized by a suitable capping agent that helps prevent unwanted agglomeration (Kundu and Liang 2008, Kundu et al. 2009, Mandal et al. 2003, Jana et al. 2001, Chen et al. 2007, Kundu et al. 2008). The synthesis of transition metal oxides with well-controlled size and shape remains a challenging task.

Among the binary transition metal oxides, cobalt is particularly interesting because of its multiple oxidation states, e.g. $\mathrm{CoO}$ and $\mathrm{Co}_{3} \mathrm{O}_{4}$. It is used as a catalyst precursor (Bezemer et al. 2006), for making pigments (Feldmann 2001), in lithium-ion battery materials, and in solid state sensors as an anisotropy source for magnetic recording (Skumryev et al. 2003). Moreover, CoO NPs exhibit super-paramagnetism or low temperature ferromagnetism where as bulk $\mathrm{CoO}$ is antiferromagnetic (Silva et al. 2010). Various methods like hydrothermal process (Shao et al. 2009), thermal decomposition (Liu et al. 2008), sol-gel (Sinko et al. 2011), electrochemical deposition (Meng et al. 2011), and spray-pyrolysis (Jana et al. 2011) have been used to fabricate $\mathrm{CoO}$ nanoparticles (NPs). It is difficult to prepare pure $\mathrm{CoO}$ due to surface oxidation of NPs that generates a mixture of $\mathrm{Co}_{3} \mathrm{O}_{4}$ or Co metal with $\mathrm{CoO}$.

Several groups report synthesis of cobalt nanoparticles (Yin et al. 1997, Zhang et al. 2008, Ghosh et al. 2005, Risbud et al. 2005, Park et al. 2004, Liu et al. 2006, Verelst et al. 1999, Jana et al. 2004, Do et al. 2005, Zhan et al. 2003, Yang et al. 2010). The magnetic properties of CoO NPs were also studied by several groups (Wdowik et al. 2008, Dutta et al. 2008). Wdowik et al. have stated that cation vacancies can account for the ferromagnetic properties of $\mathrm{CoO}$ based on

first principle calculations (Wdowik et al. 2008). Dutta et al. studied the ferromagnetic property of pure $\mathrm{CoO}$ NPs after annealing the precursor under $\mathrm{N}_{2}$ for 6 hours (Dutta et al. 2008). Most methods mentioned above require harsh reduction conditions, toxic chemicals, and high temperature, which produces a mixture of multiple shaped particles with low yields. Moreover, 
some of the processes produce a mix of different oxides of cobalt because special synthesis requirements are necessary to force cobalt metal to a low oxidation state. In such suitable synthetic approaches for the formation of $\mathrm{CoO}$ nanostructures are extremely desirable. Recently, UV-irradiation techniques have been exploited to prepare metal NPs of definite sizes and shapes (Kundu and Liang 2009, Kundu et al. 2010). However, the synthesis of oxide NPs, mainly CoO NPs using UV-irradiation techniques has only begun to be explored. The details of synthesis process, chemicals and instruments used for their characterization are discussed in the supporting information section.

In the present research, we synthesized shape-selective CoO NPs of various structures, such as nanospheres, nano-rings, nano-flowers, and nanowires of multiple sizes, by controlling the concentration of metal-ion-to-surfactant molar ratios and changing other reaction parameters. Characterization using X-ray photoelectron spectroscopy for Co 2p, 3p and 3s core-levels shows the paramagnetic high-spin Co(II) electronic configuration. The ferromagnetic and paramagnetic properties were found of $\mathrm{CoO}$ of different nanostructures.

\section{Results and Discussion}

Shape-selective CoO NPs were prepared by reduction of Co(II) ions using alkaline 2,7DHN under 4 hour of UV photo-irradiation. The details synthesis process, chemical and instruments used, characterization of the $\mathrm{CoO}$ NPs are discussed in the supporting information. When the reaction mixture (containing CTAB, 2,7-DHN, $\mathrm{NaOH}$, and $\mathrm{Co}$ (II) ions) is UV photoirradiated, the color changes from light blue to nearly black (after 4 hours) with increasing irradiation time which signifies the progress of reduction. The successive change in color with time was shown in scheme 1. The change of color is either due to change of coordination of the metal ion or due to formation of complex in solution. Figure 1 shows the UV-vis spectrum of the reaction mixture at different stages of the synthesis process. Figure 1, curve A is the absorption

band for only $\mathrm{Co}(\mathrm{II})$ ions in aqueous solution (light pink) due to formation of the $\left[\mathrm{Co}\left(\mathrm{H}_{2} \mathrm{O}\right)_{6}\right]^{2+}$ complex. The broad band comes in the 500-550 nm regions. Figure 1, curve B shows the absorption spectra of aqueous CTAB solution, which has no specific band in the UV-vis region. Aqueous 2,7-DHN (colorless solution) shows two distinct absorption bands peaking at $281 \mathrm{~nm}$ and $320 \mathrm{~nm}$ respectively due to the aromatic moiety in the 2,7-DHN skeleton (curve C, Figure 1). Curve D, Figure 1 is the absorption spectra of the mixture of $\mathrm{Co}(\mathrm{II})$ with $\mathrm{CTAB}$ having no 
specific bands in UV-vis region. Curve $\mathrm{E}$ is the absorption band of the mixture containing $\mathrm{Co}(\mathrm{II})$, CTAB and 2,7-DHN and shows a slight shift and increase in absorption value compare to only 2,7-DHN (curve C, Figure 1) due to interaction of 2,7-DHN with others. Addition of $\mathrm{NaOH}$ to the reaction mixture containing $\mathrm{Co}(\mathrm{II}), \mathrm{CTAB}$ and 2,7-DHN resulted in a new absorption peak at $345 \mathrm{~nm}$ (curve F, Figure 1) due to change in co-ordination of metal ion or formation of other complexes in alkaline solution. The color of the reaction mixture becomes greenish due to formation of hydroxyl or quinone derivatives of 2,7-DHN in alkaline media under UV photoirradiation. After continuous 4 hour UV photo-irradiation of the solution mixture containing $\mathrm{Co}(\mathrm{II}), \mathrm{CTAB}, 2,7-\mathrm{DHN}$ and $\mathrm{NaOH}$, the color of the solution changes to deep greenish black. After centrifugation for $30 \mathrm{~min}$ at $6000 \mathrm{rpm}$ and re-dispersion in DI water, the solution became light brown in color containing CoO NPs. After second centrifugation at $4000 \mathrm{rpm}$ for $15 \mathrm{~min}$ and re-dispersion in water, the aqueous solution shows a broad absorption band peaking in the region 350-450 nm due to formation of CoO NPs (curve G, Figure 1). Inset shows the camera image of $\mathrm{CoO}$ NPs solution in water. This absorption band is consistent with other reports (Athawale et al. 2010, Kim et al. 2005). This absorption band of $\mathrm{CoO}$ shifted when the shape of the particle changed. Figure 2 shows the different absorption bands of shape-selective CoO NPs. The absorption band for $\mathrm{CoO}$ nano wires shows a broad peak at $600 \mathrm{~nm}$ for short nanowires and $646 \mathrm{~nm}$ for long nanowires. Similarly, the CoO nanoflowers also show a broad band in the 455$680 \mathrm{~nm}$ regions. The peaks near lower wavelength side, mostly below $400 \mathrm{~nm}$, are due to an excitonic absorbance band with a tail extending towards a longer wavelength because of the quantum effects. While particle shape changes from spherical to anisotropic, a new band at higher wavelength side appears = as discussed above mainly due to their change in size that inturn governed by their quantum size effects. Keng et al. (Keng et al. 2009) also observed similar absorption band for cobalt oxide NPs at longer wavelengths regions.

Figure 3 A-F are transmission electron microscopy (TEM) images of shape-selective $\mathrm{CoO}$ NPs at various reaction conditions given in Table 1. Figure 3A is the TEM image of spherical shaped $\mathrm{CoO}$ NPs at lower magnification. The inset of Figure 3A shows the much higher magnified image of same $\mathrm{CoO}$ nano spheres. The average diameters of these particles are $\sim 80 \pm 10 \mathrm{~nm}$. The highly magnified image shows that the spherical particles are aggregations of much smaller spherical particles having diameters of $\sim 5 \pm 0.7 \mathrm{~nm}$. Figure $3 \mathrm{~B}$ and $3 \mathrm{C}$ show the $\mathrm{CoO}$ NPs having a ring-like structure. From the image, the approximate diameters of the nano- 
rings are $\sim 25 \pm 5 \mathrm{~nm}$. Figure $3 \mathrm{C}$ shows a single nano-ring structure. Figure 3D shows the low magnified TEM image of CoO NPs having flower-like shapes and observed that the nominal length of the $\mathrm{CoO}$ nanoflowers are about $350 \pm 50 \mathrm{~nm}$. Inset of figure 3D shows the corresponding higher magnified image of a single nano-flower. The diameter of the nano-flower is $\sim 270 \pm 50 \mathrm{~nm}$ which can vary with the direction of measurement and the typical width of the nano-flower is $0.2 \mu \mathrm{m}$. The stabilizing molecule CTAB capped the NPs are easily noticeable. Figure $3 \mathrm{E}$ and $3 \mathrm{~F}$ show the needle shaped shorter and longer $\mathrm{CoO}$ nano wires. From these images, the average length of the shorter nanowires is $\sim 210 \pm 10 \mathrm{~nm}$ with an average diameter of $\sim 45 \pm 5 \mathrm{~nm}$ whereas the average length of the longer nanowires is $300 \pm 10 \mathrm{~nm}$ and average diameter is $\sim 35 \pm 3 \mathrm{~nm}$. Inset of Figure $3 \mathrm{E}$ and $3 \mathrm{~F}$ shows their corresponding higher magnified images. From all these TEM images, we conclude that shape-selective CoO NPs are formed at high yields by changing the concentration of reaction parameters.

Analysis of energy dispersive x-ray spectroscopic (EDS) study shows that the NPs are $\mathrm{CoO}$ with small quantities of $\mathrm{Br}$ and $\mathrm{C}$ remaining from the CTAB surfactant (Figure 4). The EDS spectra clearly indicate that CoO NPs are formed and surface is capped by the surfactant CTAB. The powder X-ray diffraction (XRD) patterns of shape-selective CoO NPs are shown in Figure 5 where (A) is for spherical NPs, (B) for nano-rings, (C) for nano-flowers, (D) and (E) are for the needle shaped short and long nanowires. The typical diffraction of CoO NPs exhibit diffraction in the $2 \theta$ range $15-80^{\circ}$ corresponding to diffraction from (111), (220), (311), (222), (422), (511), and (440) lattice planes with rock-salt structure (space group Fm3m). No diffraction peaks for $\mathrm{Co}_{3} \mathrm{O}_{4}$ or metallic $\mathrm{Co}$ was observed which confirms the formation of pure CoO NPs in the bulk. The peak positions matches with previous reports (Park et al. 2004, Yang et al. 2010, Lagunas et al. 2008, Zhang et al. 2002). Lagunas et al. (Lagunas et al. 2008) observed a broader XRD pattern in their study due to formation of very small CoO NPs. They claim that when particle size is smaller than $3 \mathrm{~nm}$, the interface phenomenon that underlies X-ray crystal diffraction starts disappearing. Park et al. (Park et al. 2004) observed that pencil shaped CoO nanocrystals can show a wurtzite structure. They also discussed that anisotropic crystal growth along $\mathrm{C}$ axis leads to the formation of pencil shaped $\mathrm{CoO}$ nanorods. In our synthesis we used CTAB as a capping agent and the selective interactions of CTAB with different crystal planes of the cobalt might alter the growth rate and produces different shaped CoO NPs. 
Figure 6 presents the XPS spectra of shape-selective CoO NPs. Analysis was performed to investigate the cobalt oxidation state of each morphology (nanoflake, nanoflower, nanorod etc.) Figure 6A shows the XPS survey spectra of the CoO NPs with the XPS and Auger lines from the constituent elements identified. For nano rod samples we also observed a small $\mathrm{N} 1 \mathrm{~s}$ peak at $399.50 \mathrm{eV}$. The $\mathrm{C}$ and $\mathrm{N}$ peak originated from the capping agent, CTAB used for our synthesis. Figure $6 \mathrm{~B}$ shows the high-resolution Co $2 \mathrm{p}_{3 / 2,1 / 2}$ core-level spectra for the three different shaped CoO NPs. The Co $2 \mathrm{p}_{3 / 2,1 / 2}$ spin-orbit separation is $15.9 \mathrm{eV}$ for all the samples and they have satellite structures $6 \mathrm{eV}$ above the $\mathrm{Co} 2 \mathrm{p}$ peaks indicative of high spin $\mathrm{Co}^{2+}$ ions. The largest influence on satellite structure is due to charge-transfer between the Co $d$ states and the $\mathrm{O} p$ ligand states, and thus the shakeup satellite peaks are indicative of the paramagnetic high-spin $\mathrm{Co}(\mathrm{II})$ electronic configuration $\left[2 \mathrm{p}^{5} 3 \mathrm{~d}^{7}\right]$ in $\mathrm{CoO}$ NPs. These binding energies and satellite structures are well matched with previous literature reports (Ghosh et al. 2005, Yang et al. 2010, Li et al. 2009). The absence of a peak at $778.1 \mathrm{eV}$ signifies that there are no Co metal impurities consistent with XRD results. Figure 6C shows the Co 3s and 3p core-level spectra for the three different shaped $\mathrm{CoO}$ NPs. The binding energy of the Co $3 p$ peak centroid is $60.6 \mathrm{eV}$ for each morphology and thus exhibits no chemical shift. The strong coupling between the $3 p$ core hole in the final state and the partially filled $3 \mathrm{~d}$ orbitals yield the observed multiplets $\left[3 \mathrm{p}^{6} 3 \mathrm{~d}^{7}\right.$ to $\left.3 \mathrm{p}^{5} 3 \mathrm{~d}^{8}\right]$. Note that the spectra for $\mathrm{CoO}$ nanoflower and $\mathrm{CoO}$ nanowire have a stronger than usual feature $7.2 \mathrm{eV}$ from the main peak. Also, the broad feature $\approx 20 \mathrm{eV}$ from the main $3 \mathrm{p}$ peak in all the spectra is attributed to an energy-loss peak. For Co dihalides, the spectral splitting of the $3 \mathrm{~s}$ peak mostly represents the $3 \mathrm{~s}-3 \mathrm{~d}$ exchange splitting of the $3 \mathrm{~s}^{1} 3 \mathrm{~d}^{8}$ configuration. Since the Co 3s peaks for each morphology exhibit a multiplet structure, the data provides further evidence for the presence of the high-spin Co(II) configuration. We did some control experiments to study various reaction parameters for the formation of shape-selective $\mathrm{CoO}$ NPs and the mechanism of particle formation that are discussed below.

For the formation of shape-selective $\mathrm{CoO}$ NPs we carried out some control experiments by altering the concentration of $\mathrm{Co}(\mathrm{II})$ ions, 2,7-DHN, CTAB and $\mathrm{NaOH}$. We also varied UVirradiation exposure time. The shape-selective $\mathrm{CoO}$ NPs are formed at a particular concentration that is given in Table 1 in main text which illustrates that spherical CoO NPs form at low surfactant concentration whereas other shapes like nano-rings, nano-flowers or nanowires form at higher concentration of the surfactant. When we reduced the $\mathrm{Co}$ (II) ion concentration to $\leq 2 \times$ 
10-5 M, a longer UV-irradiation time is required to complete the reaction. When the $\mathrm{Co}(\mathrm{II})$ ion concentration is high $(\sim 10-1 \mathrm{M})$, the solution becomes hazy and a mixture of different shaped particles forms. When we dramatically increase the concentration of reducing agent (2,7-DHN) $(\geq 0.5 \times 10-1 \mathrm{M})$, the formation of NPs starts very fast but precipitates in the solution. Similarly, at low concentrations of 2,7-DHN $(\leq 10-6 \mathrm{M})$, a longer UV-irradiation time needed to start particle formation. We also examined $\mathrm{NaOH}$ concentration. At very high concentration, mixed undefined shaped particles form but mostly precipitate and agglomerate after few hours. When testing the UV photo-irradiation time, 4 hours were sufficient for the formation of shapeselective CoO NPs. Shorter UV-irradiation times (1-2 hours) produced partially grown particles with undefined shapes whereas longer irradiation (8-10 hours) produced multiple shaped aggregated particles. The average particle sizes, their corresponding shapes, and distribution are given in Table 1 in the main text. Thus all these control experiments prove that reagent concentration is extremely important for the formation of shape-selective CoO NPs with well defined morphology.

The formation mechanisms of shape-selective CoO NPs have been studied in details. To control the shape of CoO NPs, the presence of CTAB and 2,7-DHN are important. In the absence of CTAB, $\mathrm{CoO}$ precipitates as micron sized particles of undefined shape within a short time. In the absence of 2,7-DHN, no NPs form due to the lack of a reducing agent. The presence of $\mathrm{NaOH}$ enhances the reducing power of 2,7-DHN and leads to the formation of anisotropic NPs with fascinating shapes. Most of the hydroxyl compounds like ascorbic acid (Pal and Pal 1999), TX-100 (Pal 1998), and poly (vinyl) alcohol (Henglein 1999) undergo hydroxylic cleavage to produce radical species in presence of UV photo-irradiation. This radical species acts as a reducing agent for the reduction of metal ions to metal ${ }^{0}$. We have reported previously that hydroxylic compounds reduce $\mathrm{Ag}(\mathrm{I})$, $\mathrm{Au}(\mathrm{III})$ etc., to produce their corresponding NPs with various morphology.4Moreover, we also reported that deoxyribonucleic acid (DNA) having hydroxylic group on its sugar part also acts as a reducing agent for the synthesis of metal nanowires (Kundu and Liang 2008). In the present work, we believe that a radical species is generated and is responsible for the reduction of $\mathrm{Co}$ (II) ions. To confirm this claim, we performed some control experiments. UV photo-irradiation was conducted at $270 \mathrm{~nm}$ which is very close to the absorbance of 2,7-DHN. When we irradiate the reaction mixture far below ( $180 \mathrm{~nm})$ or far above $(\sim 370 \mathrm{~nm})$ the absorption band of 2,7-DHN, did not result the formation 
of $\mathrm{CoO}$ on a time scale of 4 hours. This experiment supports the argument that radical species are responsible for the reduction of $\mathrm{Co}$ (II) ions. At ambient condition, the as prepared Co particles are not stable and immediately oxidize to produce CoO NPs. At the Initial reaction stage, the Co particles nucleate to form Co seed particles and with time they aggregate to form bigger particles. Once the reduction of Co(II) starts, the growth of the particles takes place in several steps in presence of CTAB and generates definite shapes. According to previous reports, the specific shape of particles mainly depends on two parameters: the faceting tendency of the stabilization agent and the growth kinetics of metal, i.e., the rate of supply of metal (0) to the different crystallographic planes (Petroski et al. 1998).6 As summarized in Table 1, at low concentration of CTAB, spherical particles are formed whereas at high concentrations the more anisotropic shapes form. Murphy's group reported that CTAB capped Au nanorods and nanowires form mostly at higher concentrations $(\sim 0.1 \mathrm{M})$ of surfactant. The formation of $\mathrm{CoO}$ nano-rings or nano-flowers took place at a medium concentration range of CTAB. Scheme 2 shows the formation of $\mathrm{CoO}$ NPs with varying reaction conditions. A proper concentration of CTAB play an important role for the formation and stabilization of the NPs in aqueous solution as reported earlier. A clear example of this behavior can be found for the formation of cobalt NPs whose crystalline phase (Dinega et al. 1999), shape (Puntes 2002) and direct mechanism of formation are controlled by the surfactant. Moreover, as we discussed earlier, with the increasing time of irradiation the color of the solution changes as we can see from the scheme 1 (main text) which is a good indication for the progress of the reaction. Similar types of color change have been observed (Nam et al. 2010) for the formation of $\mathrm{CoO}$ nanostructures with different morphology. Further studies will focus on optimizing the synthesis process making such structures and their corresponding magnetic properties will be further investigated.

The magnetic measurements were performed on batches of spheres and rings as a function of temperature and applied magnetic field. The spheres contain at least two phases, the majority phase displays Curie like paramagnetism for all measured temperatures $(\mathrm{T}>2 \mathrm{~K})$ with a small second phase that is ferromagnetic (FM) illustrated in Figure 7a. The FM component is more clearly observable in the inset of Figure $7 \mathrm{a}$ which shows $\mathrm{M}(\mathrm{H})$ for isotherms at $5 \mathrm{~K}, 30 \mathrm{~K}$, and $150 \mathrm{~K}$ where a linear correction has been applied to each trace to remove a small diamagnetic contribution.. The $5 \mathrm{~K}$ data illustrates that the paramagnetic contribution dominates at low temperatures and high magnetic fields. Consistent with the small ferromagnetic signal, a 
hysteresis loop with a coercive field of $\sim 100$ Oe is observed at all measured temperatures indicative of bulk Co. Given the constituent materials, the only magnetic atoms are Co and thus both behaviors are expected to arise from Co atoms, with the FM signal from small particles of metallic Co and the Curie behavior from isolated Co ions or sub-nanometer clusters of Co. The zero temperature saturation moment for ferromagnetic $C_{0}$ is $\mathrm{M}_{\mathrm{o}}=1.44 \times 10^{6} \mathrm{~A} / \mathrm{m}($ Coey 2010.) and the effective moment for the paramagnetic Co ions is $p_{\text {eff }}=4.8 \mu_{\mathrm{B}}$, so this implies that the ferromagnetic component accounts for less than ten percent of the Co atoms.

The nano-rings are far more interesting magnetically. Figure $7 \mathrm{~b}$ shows zero-field cooled (ZFC) and field cooled (FC) measurements in a 10 Oe applied field. The ZFC curve shows a distinct peak at $6 \mathrm{~K}$, while the $\mathrm{FC}$ curve saturates at low temperature. These curves are consistent with FM behavior below 6K, which may be attributed to superparamagnetic Co particles. Superparamagnetism occurs when the magnetic energy $(\mathrm{KV}$, where $\mathrm{K}$ is the magnetocrystalline anisotropy and $\mathrm{V}$ is the particle volume) of tiny ferromagnetic particles is comparable to the thermal energy of the system. The individual particles remain ferromagnetic with the spins locked together, but the orientation of the total moment fluctuates with a time constant given by:

$$
\tau=\frac{1}{f_{o}} \exp \left(\frac{K V}{k_{B} T}\right)
$$

where $f_{\mathrm{o}}$ is the attempt frequency, typically assumed to be $\sim 10^{10} \mathrm{~Hz}$. From a measurement perspective, particles will appear magnetically stable for scales where $\tau$ is large relative to the measurement time. For typical magnetization measurements this corresponds to a few seconds, making $\mathrm{KV} \sim 25 \mathrm{k}_{\mathrm{B}} \mathrm{T}$. The magnetic anisotropy energy (at $0 \mathrm{~K}$ ) of FCC Co is $\mathrm{K}=6.8 \times 10^{4} \mathrm{~J} / \mathrm{m}^{3}$. Using $6 \mathrm{~K}$ as a blocking temperature results in a typical particle diameter of $3 \mathrm{~nm}$ for spherical particles. While the rings are not spherical, this result indicates that the individual rings are made up of aggregates of much smaller particles which are not magnetically coupled to each other. The inset of Figure $7 \mathrm{~b}$ shows the isothermal magnetization curves for temperatures below, at, and above the blocking temperature. The $2 \mathrm{~K}$ measurement shows a coercivity of $\sim 500$ Oe, while the curves at $6 \mathrm{~K}$ and $10 \mathrm{~K}$ show no indication of hysteresis as expected for particles above the blocking temperature. This superparamagnetic behavior is intrinsic to the small size of the fundamental cobalt nanoparticles which are chemically self-assembled to produce ring structures. The nano-flowers and nanowires will be magnetically investigated in future work. 


\section{Conclusion}

, We have successfully synthesized CoO NPs under UV photo-irradiation with a one-step process. Distinct shapes including nanospheres, nano-rings, nano-flowers, and nanowires were obtained by controlling the concentration of CTAB and alkaline 2,7-DHN and changing the other reaction parameters. The magnetic measurements indicate that the nano-rings are superparamagnetic with a $6 \mathrm{~K}$ blocking temperature while the nanospheres remain paramagnetic. XPS results indicate the paramagnetic high-spin Co(II) electronic configuration. The shapeselective $\mathrm{CoO}$ NPs could be used for applications like sensors, catalytic fields, information storage, and electrode materials, among many others.

Supporting Information Available: Synthesis details, chemicals used, instruments used for the shape-controlled synthesis of CoO NPs are provided.

Acknowledgement: This research was in part sponsored by the NSF-0506082; the Department of Mechanical Engineering, Texas A\&M University; and the Texas Engineering Experiments Station. Supports for TEM and EDS by Dr. Zhiping Luo at the Microscopy Imaging Center (MIC), Texas A\&M University were greatly appreciated. This work performed under the auspices of the U.S. Department of Energy by Lawrence Livermore National Laboratory under Contract DE-AC52-07NA27344.

\section{References:}

Alivisatos A P (1996) Semiconductor Clusters, Nanocrystals, and Quantum Dots. Science 271: 933-937.

Athawale A A, Majumdar M, Singh H' Navinkiran K (2010) Synthesis of Cobalt Oxide Nanoparticles/Fibres in Alcoholic Medium using g-ray Technique Defence Sci. J. 60: 507-513. 
Bezemer G L, Bitter J H, Kuipers H P C E, Oosterbeek H, Holewijn J E, Xu X, Kapteijn F, van

Dillen A J, de Jong K P (2006) Cobalt Particle Size Effects in the Fischer-Tropsch Reaction Studied with Carbon Nanofiber Supported Catalysts. J. Am. Chem. Soc. 128: 3956-3964.

Chen Y, Johnson E, Peng X (2007) Formation of Monodisperse and Shape-Controlled MnO Nanocrystals in Non-Injection Synthesis: Self-Focusing via Ripening. J. Am. Chem. Soc. 129: 10937-10947.

Coey JMD, (2010) Magnetism and Magnetic Materials, Cambridge University Press, New York.

Dinega D P, Bawendi M G (1999) A Solution-Phase Chemical Approach to a New Crystal Structure of Cobalt. Angew. Chem., Int. Ed. 38: 1788-1791.

Do J S, Weng C H (2005) Preparation and characterization of $\mathrm{CoO}$ used as anodic material of lithium battery. J. Power Sources 146: 482-486.

Dutta D P, Sharma G, Manna P K, Tyagi A K, Yusuf S M (2008) Room temperature ferromagnetism in $\mathrm{CoO}$ nanoparticles obtained from sonochemically synthesized precursors. Nanotechnology 19: 245609-245615.

Feldmann C, Jungk H -O (2001) Polyol-Mediated Preparation of Nanoscale Oxide Particles. Angew. Chem., Int. Ed. 40: 359-362.

Ghosh M, Sampathkumaran E V, Rao C N R (2005) Synthesis and Magnetic Properties of CoO Nanoparticles. Chem. Mater. 17: 2348-2352. 
Henglein A (1999) Radiolytic preparation of ultrafine colloidal gold particles in aqueous solution: optical spectrum, controlled growth, and some chemical reactions. Langmuir 15: 67386744.

Jang H, Seong C, Suh Y, Kim H, Lee C (2011) Synthesis of Lithium-Cobalt Oxide nanoparticles by Flame Spray Pyrolysis. Aerosol Sci. and Technol. 38: 1027-1032.

Jana N R, Chen Y, Peng X (2004) Size- and Shape-Controlled Magnetic (Cr, Mn, Fe, Co, Ni) Oxide Nanocrystals via a Simple and General Approach. Chem. Mater. 16: 3931-3935.

Jana N R, Gearheart L, Murphy C J (2001) Wet Chemical Synthesis of High Aspect Ratio Cylindrical Gold Nanorods. J. Phys. Chem. B 105: 4065-4067.

Keng P Y, Kim B Y, Shim I, Sahoo R, Veneman P E, Armstrong N R. Yoo H, Pemberton J E, Bull M M, Griebel J J, Ratcliff E L, Nebesny K G, Pyun J (2009) Colloidal polymerization of polymer-coated ferromagnetic nanoparticles into cobalt oxide nanowires. ACS Nano 3: 31433157.

Kim J -W, Choi S H, Lillehei P T, Chu S -H, King G C, Watt G D (2005) Cobalt oxide hollow nanoparticles derived by bio- templating. Chem. Commun. 4101-4103.

Kundu S, Liang H (2010) Photoinduced formation of shape-selective Pt nanoparticles. Langmuir 26: 6720-6727.

Kundu S, Lau S, Liang H (2009) Shape-Controlled Catalysis by Cetyltrimethylammonium Bromide Terminated Gold Nanospheres, Nanorods, and Nanoprisms. J. Phys. Chem. C 113: 5150-5156. 
Kundu S, Wang K, Liang H (2009) Photochemical Generation of Catalytically Active Shape Selective Rhodium Nanocubes. J. Phys. Chem. C 113: 18570-18577.

Kundu S, Liang H (2008) Photochemical Synthesis of Electrically Conductive CdS Nanowires on DNA Scaffolds. Adv. Mater. 20: 826-831.

Kundu S, Peng L, Liang H (2008) A new route to obtain high-yield multiple-shaped gold nanoparticles in aqueous solution using microwave irradiation. Inorg. Chem. 47: 6344-6352.

Kundu S, Wang K, Liang H (2009) Size-controlled synthesis and self-assembly of silver nanoparticles within a minute using microwave irradiation. J. Phys. Chem. C 113: 134-141.

Kundu S, Liang H (2008) Microwave Synthesis of Electrically Conductive Gold Nanowires on DNA Scaffolds. Langmuir 24: 9668-9674.

Lagunas A, Payeras A M, Jimeno C, Puntes V F, Pericas M A (2008) Low-Temperature Synthesis of $\mathrm{CoO}$ Nanoparticles via Chemically Assisted Oxidative Decarbonylation. Chem. Mater. 20: 92-100.

Li L, Sasaki T, Shimizu Y, Koshizaki N (2009) Controlled Cobalt Oxide from Two-Dimensional Films to One-Dimensional Nanorods and Zero-Dimensional Nanoparticles: MorphologyDependent Optical Carbon Monoxide Gas-Sensing Properties. J. Phys. Chem. C 113: 1594815954.

Liu X, Yi R, Zhang N, Shi R, Li X, Qiu G (2008) Cobalt Hydroxide Nanosheets and Their Thermal Decomposition to Cobalt Oxide Nanorings. Chem. Asian J. 3: $732-738$.

Liu J F, Yin S, Wu H P, Zeng Y W, Hu X R, Wang Y W, Lv G L, Jiang J Z (2006) Wurtzite-torocksalt structural transformation in nanocrystalline CoO. J. Phys. Chem. B 110: 21588-21592. 
Mandal M, Kundu S, Sau T K, Yusuf S M, Pal T (2003) Synthesis and characterization of superparamagnetic Ni-Pt nanoalloy. Chem. Mater. 15: 3710-3715.

Meng Z, Liu B, Zheng J, Sheng O, Zhang H (2011) Electrodeposition of cobalt oxide nanoparticles on carbon nanotubes, and their electrocatalytic properties for nitrite electrooxidation. Microchimica Acta. 175: 251-257.

Nam K M, Shim J H, Han D, Kwon H S, Kang Y, Li Y, Song H, Seo H, Seo W, Park J T (2010) Syntheses and Characterization of Wurtzite $\mathrm{CoO}$, Rocksalt $\mathrm{CoO}$, and Spinel $\mathrm{Co}_{3} \mathrm{O}_{4}$ Nanocrystals: Their Interconversion and Tuning of Phase and Morphology. Chem. Mater. 22: 4446-4454.

Pal A, Pal T (1999) Silver nanoparticle aggregate formation by a photochemical method and its application to SERS analysis. J. Raman Spectrosc. 30: 199-204.

Pal A (1998) Photoinitiated gold sol generation in aqueous Triton X-100 and its analytical application for spectrophotometric determination of gold. Talanta 46: 583-587.

Park J, An K, Hwang Y, Park J-G, Noh H-J, Kim J-Y, Park J-H, Hwang N-M, Hyeon T (2004) Ultra-large-scale syntheses of monodisperse nanocrystals. Nat. Mater. 3: 891-895.

Petroski J M, Wang Z L, Green T C, El-Sayed M A (1998) Kinetically Controlled Growth and Shape Formation Mechanism of Platinum Nanoparticles. J. Phys. Chem. B 102: 3316-3320.

Puntes V F, Zanchet D, Erdonmez C K, Alivisatos A P (2002) Synthesis of hcp-Co Nanodisks. J. Am. Chem. Soc. 124: 12874-12880.

Risbud A S, Snedeker L P, Elcombe M M, Cheetham A K, Seshadri R (2005) Wurtzite CoO. Chem. Mater. 17: 834-838. 
Sinkó K, Szabó G, Zrínyi M (2011) Liquid-Phase Synthesis of Cobalt Oxide Nanoparticles. J. Nanosci. Nanotechnol. 11: 4127-4135.

Silva N J O, Millán A, Palacio F, Martins M, Trindade T, Puente-Orench I, Campo J (2010) Remanent magnetization in $\mathrm{CoO}$ antiferromagnetic nanoparticles. Phys. Rev. B 82: 094433094440.

Shao Y, Sun J, Gao L (2009) Hydrothermal Synthesis of Hierarchical Nanocolumns of Cobalt Hydroxide and Cobalt Oxide. J. Phys. Chem. C 113: 6566-6572.

Skumryev V, Stoyanov S, Zhang Y, Hadjipanayis G, Givord D, Nogués J (2003) Beating the superparamagnetic limit with exchange bias. Nature 423: 850-853.

Verelst M, Ely T O, Amiens C, Snoeck E, Lecante P, Mosset A, Respaud M, Broto J M, Chaudret B (1999) Synthesis and characterization of $\mathrm{CoO}, \mathrm{Co}_{3} \mathrm{O}_{4}$, and mixed $\mathrm{Co} / \mathrm{CoO}$ nanoparticles. Chem. Mater. 11: 2702-2708.

Wdowik U D, Parlinski K (2008) Electronic structure of cation-deficient CoO from first principles. Phys. Rev. B 77: 115110-115112.

Yang G, Gao D, Shi Z, Zhang Z, Zhang J, Zhang J, Xue D (2010) Room Temperature Ferromagnetism in Vacuum-Annealed CoO Nanospheres. J. Phys. Chem. C 114: 21989-21993.

Yin J S, Wang Z L (1997) In situ structural evolution of self-assembled oxide nanocrystals. J. Phys. Chem. B 101: 8979-8983.

Zhang Y, Zhu J, Song X, Zhong X (2008) Controlling the synthesis of CoO nanocrystals with various morphologies. J. Phys. Chem. C 112: 5322-5327. 
Zhan Y J, Yin C R, Wang W Z, Wang G H (2003) Synthesis of CoO fibers in pyrolytic process. Mater. Lett. 57: 3402-3405.

Zhang L, Xue D J (2002) Preparation and magnetic properties of pure CoO nanoparticles. J. Mat. Sci. Lett. 21: 1931-1933.

\section{Figure Captions:}

Figure 1. The UV-vis spectrum of the reaction mixture at different stages of the synthesis process. (A) is the absorption band for only $\mathrm{Co}$ (II) ions in aqueous solution; (B) is the absorption spectra of aqueous CTAB solution; (C) is the absorption spectra of aqueous 2,7-DHN solution; (D) is the absorption spectra of the mixture of $\mathrm{Co}(\mathrm{II})$ with CTAB; (E) is the absorption band of the mixture containing $\mathrm{Co}(\mathrm{II}), \mathrm{CTAB}$ and 2,7-DHN; (F) is the absorption spectra of the mixture $\mathrm{Co}(\mathrm{II}), \mathrm{CTAB}, 2,7-\mathrm{DHN}$, and $\mathrm{NaOH}$; $(\mathrm{G})$ is the absorption band of pure $\mathrm{CoO}$ NPs. The inset shows the image of a CoO NPs solution in water.

Figure 2. Curve A to E shows the different absorption bands of shape-selective CoO NPs; (A) for spherical NPs; (B) for nano-rings; (C) for nano-flowers; (D) and (E) for the needle shaped short and long nanowires.

Figure 3. The transmission electron microscopy (TEM) images of shape-selective CoO NPs. Figure 3A shows the TEM image of spherical CoO NPs at low magnification. The inset of Figure 3A shows the much higher magnified image of same $\mathrm{CoO}$ nano spheres. Figure $3 \mathrm{~B}$ and 3C shows the low and high magnified TEM of CoO NPs having ring like structure. Figure 3D shows the TEM image of $\mathrm{CoO}$ NPs having flower like shapes and inset shows a single nano flower. Figure 3E shows the TEM image of needle shaped shorter $\mathrm{CoO}$ nano wires. Figure 3F shows the TEM images of longer sizes $\mathrm{CoO}$ nano wires. Inset of Figure 3E and 3F shows their corresponding higher magnified images. 
Figure 4. The energy dispersive x-ray spectroscopic (EDS) analysis of shape-selective CoO NPs.

Figure 5. The powder X-ray diffraction (XRD) pattern of shape-selective CoO NPs: (A) for spherical NPs; (B) for nano-rings; (C) for nano-flowers; (D) and (E) for the needle shaped short and long nanowires.

Figure 6. The X-ray photoelectron spectroscopy (XPS) of shape-selective CoO NPs. Figure 6A shows the overall XPS survey of CoO NPs. Figure $6 \mathrm{~B}$ shows the Co $2 \mathrm{p}_{3 / 2}$, Co $2 \mathrm{p}_{1 / 2}$ and Figure $6 \mathrm{C}$ shows the Co 3s, Co $3 p$ core-levels spectrum for the three different shaped CoO NPs respectively.

Figure 7. Magnetization of $\mathrm{CoO}$ NPs. Figure 7A Magnetization of spherical CoO NPs showing Curie behavior on top of a large temperature independent positive background. The inset of Figure 7a shows $\mathrm{M}(\mathrm{H})$ illustrating the ferromagnetic contribution to the NPs. Figure 7B shows zero field cooled and field cooled measurements on $\mathrm{CoO}$ nano-rings where the $6 \mathrm{~K}$ peak is the blocking temperature $\left(\mathrm{T}_{\mathrm{B}}\right)$. The inset shows the isothermal magnetization curves, with hysteresis appearing only below the $\mathrm{T}_{\mathrm{B}}$.

Table 1: The detailed final concentrations of all the reaction parameters, time of UV Photoirradiation, and particle size and shape distribution for the formation of $\mathrm{CoO}$ NPs.

Scheme 1. The successive change in color with time during the formation of CoO NPs using UV photo-irradiation.

Scheme 2. Schematic presentation for the formation of shape-selective CoO NPs. 


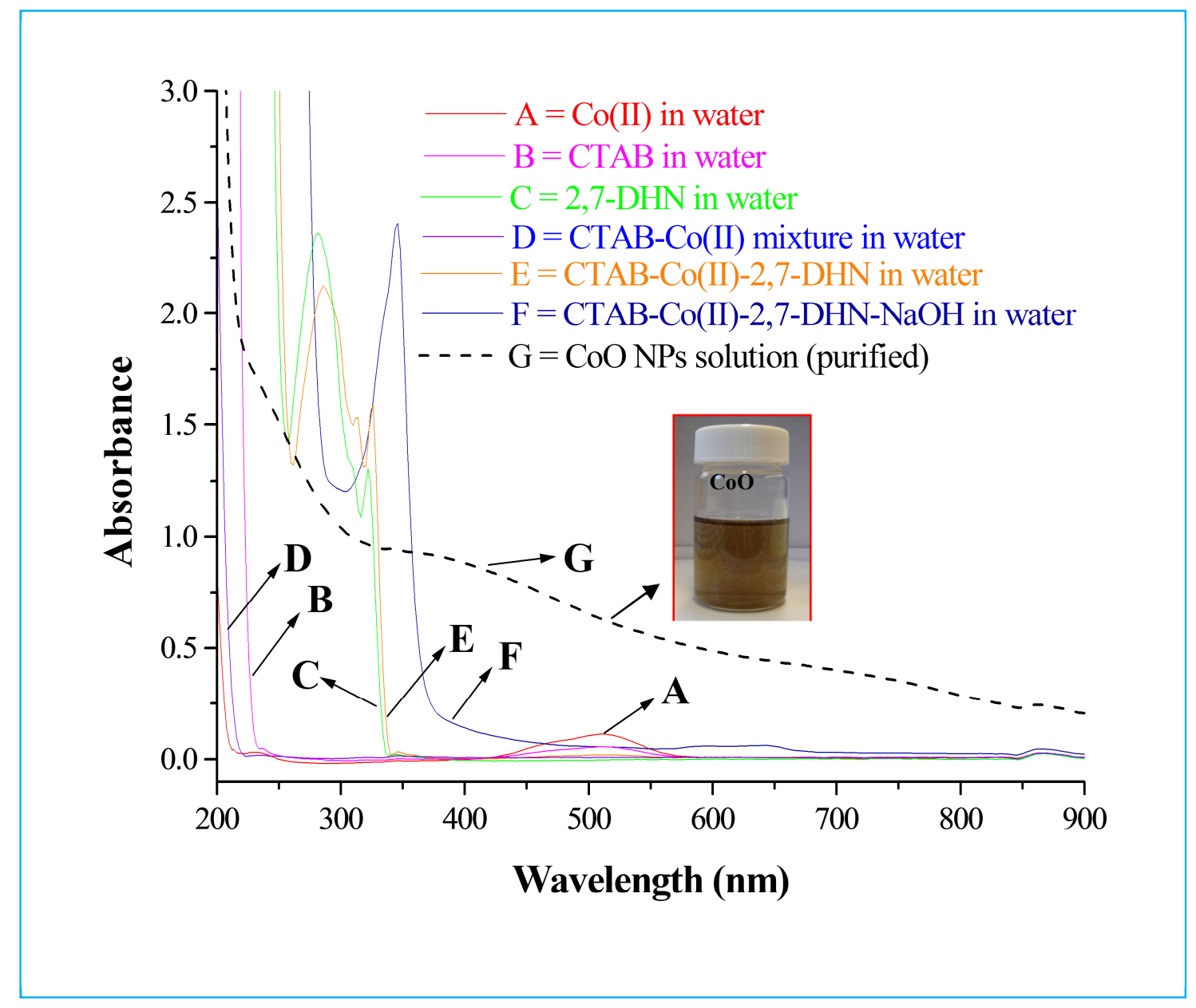

Figure 1 


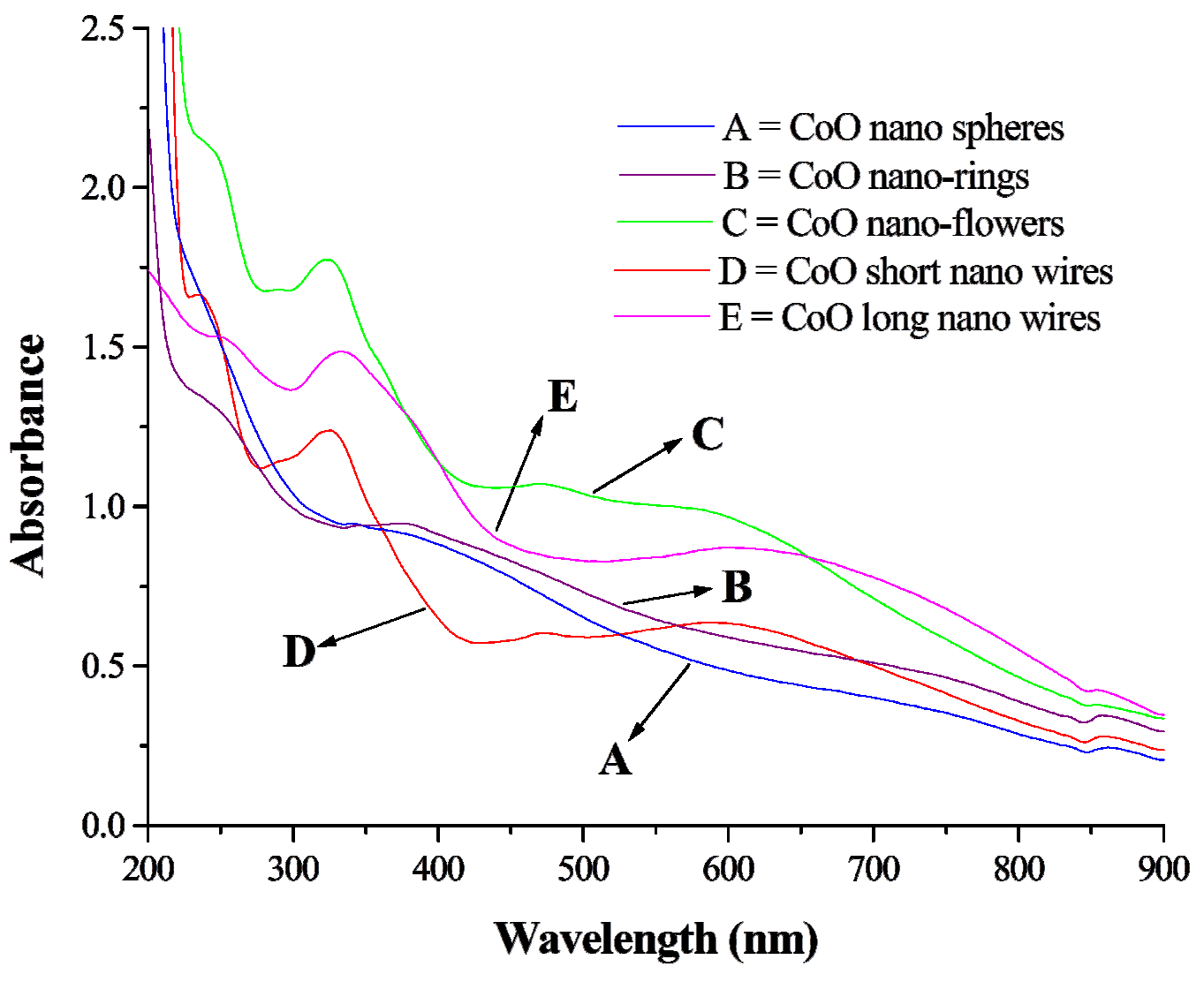

Figure 2 

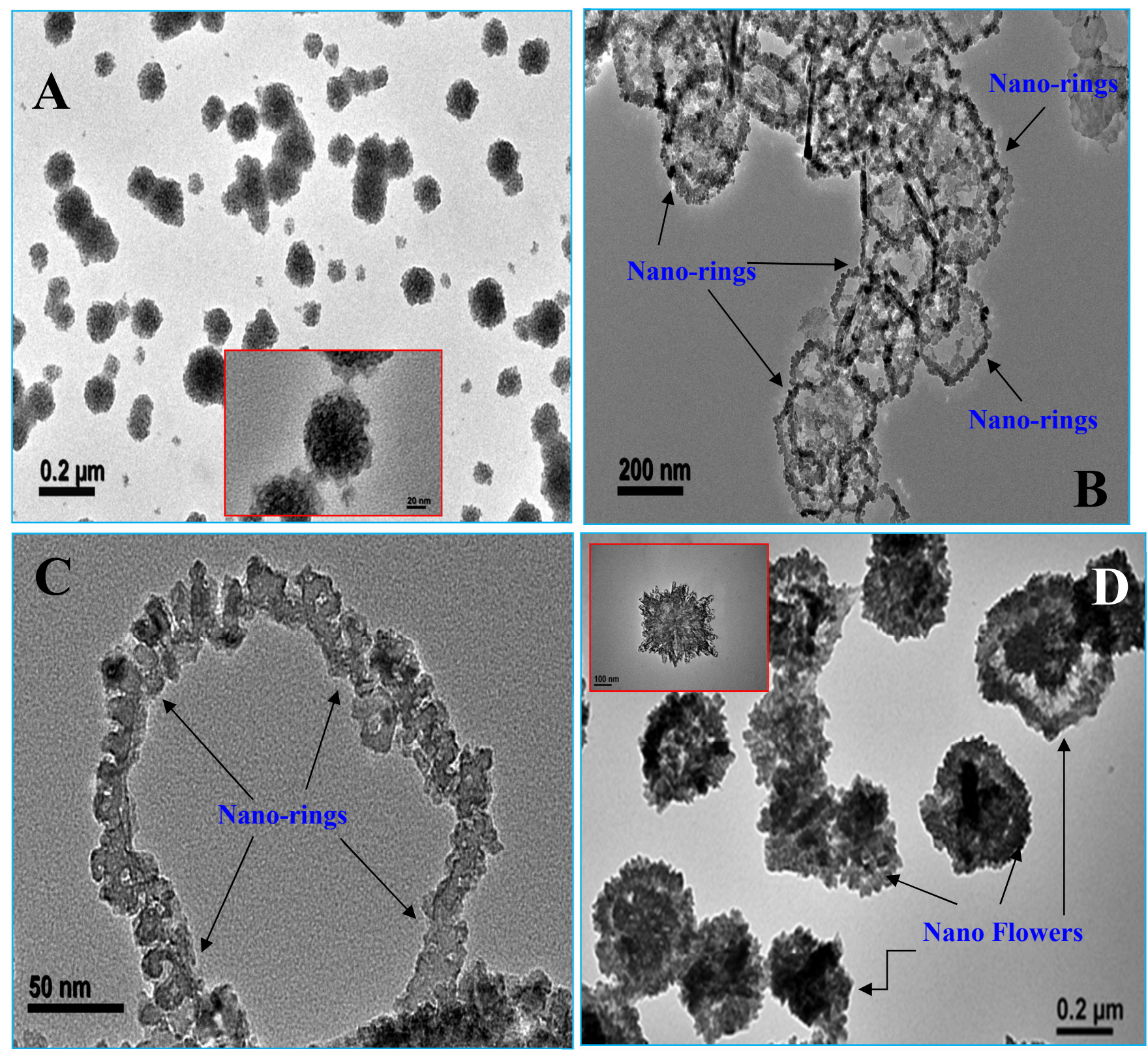

Figure 3, A, B, C, D 

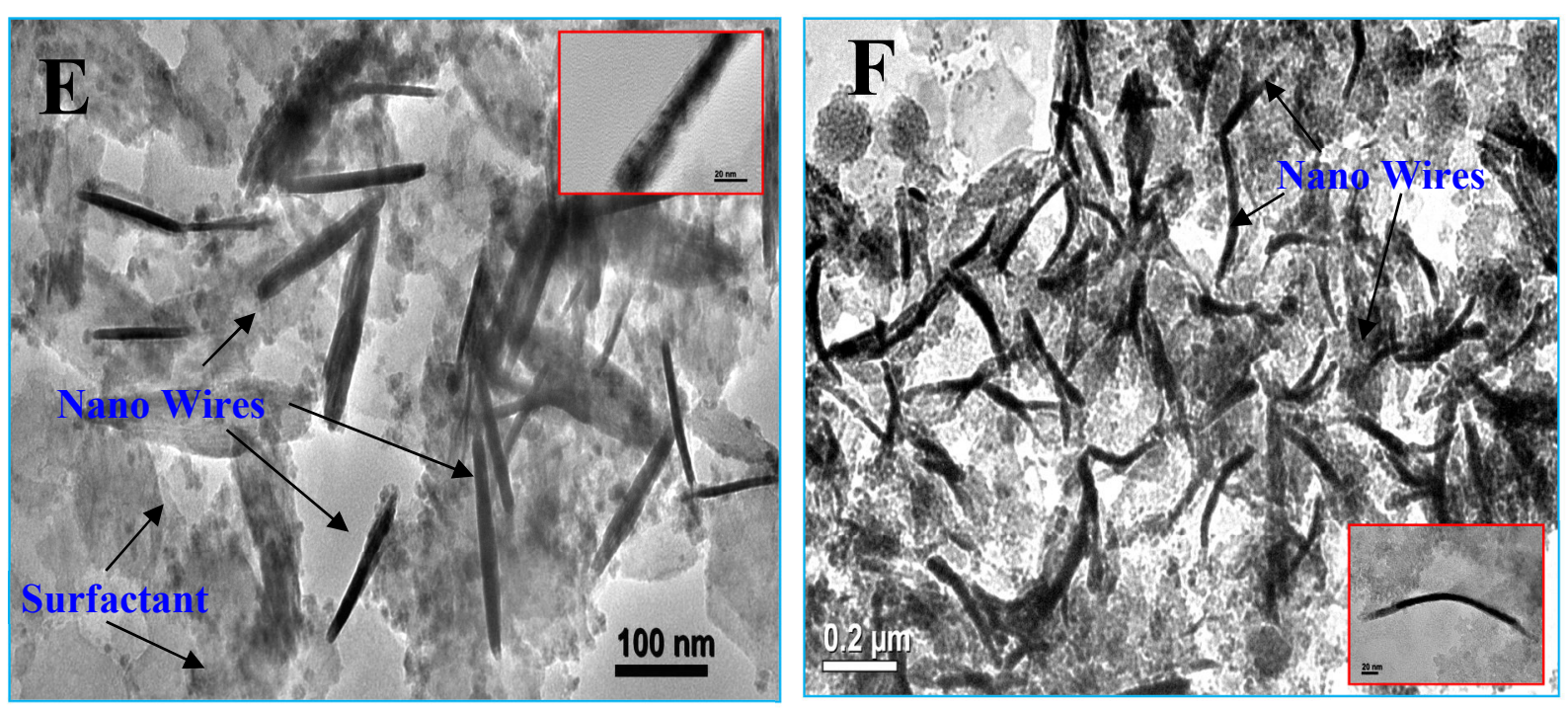

Figure 3, E-F 


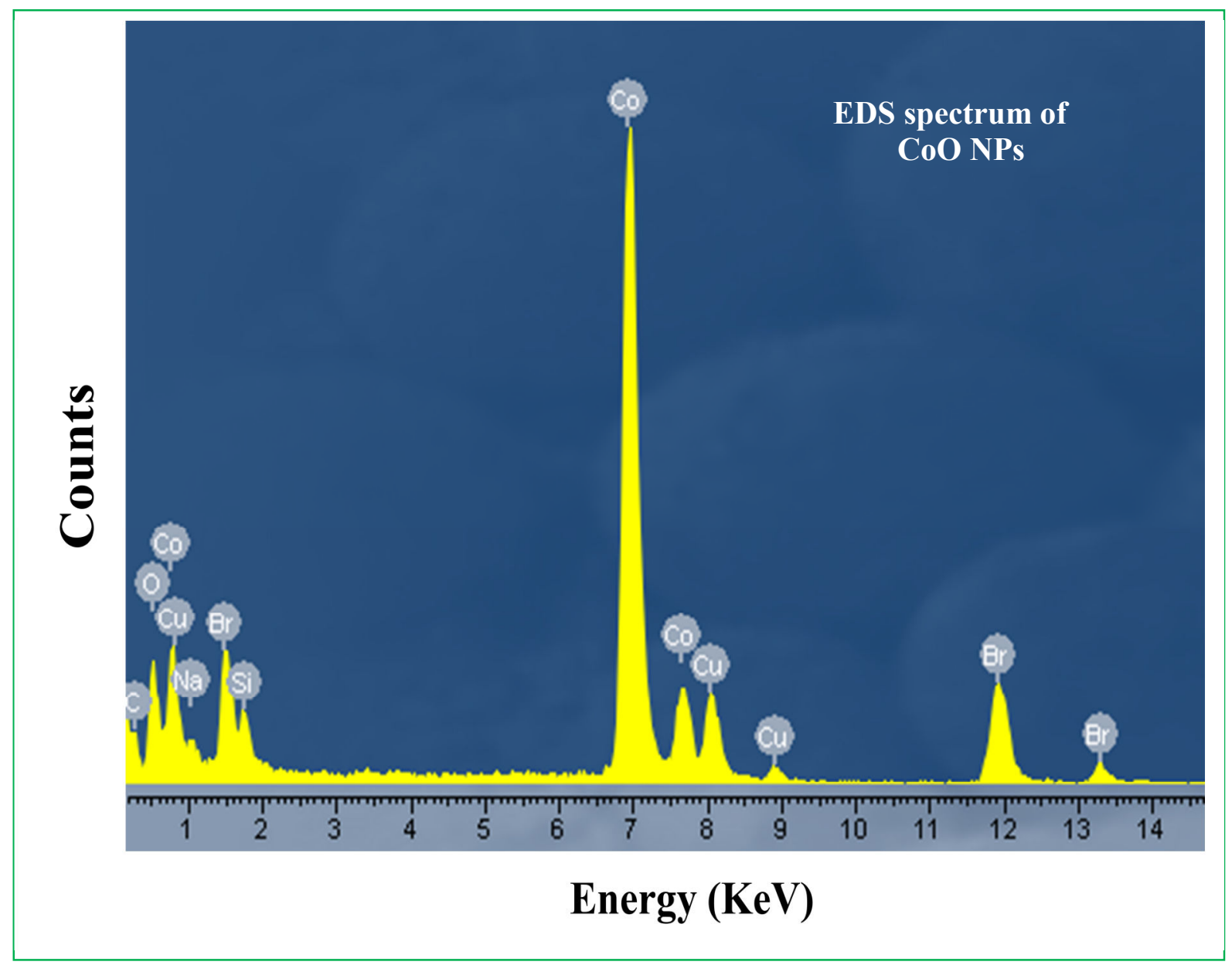

Figure 4 


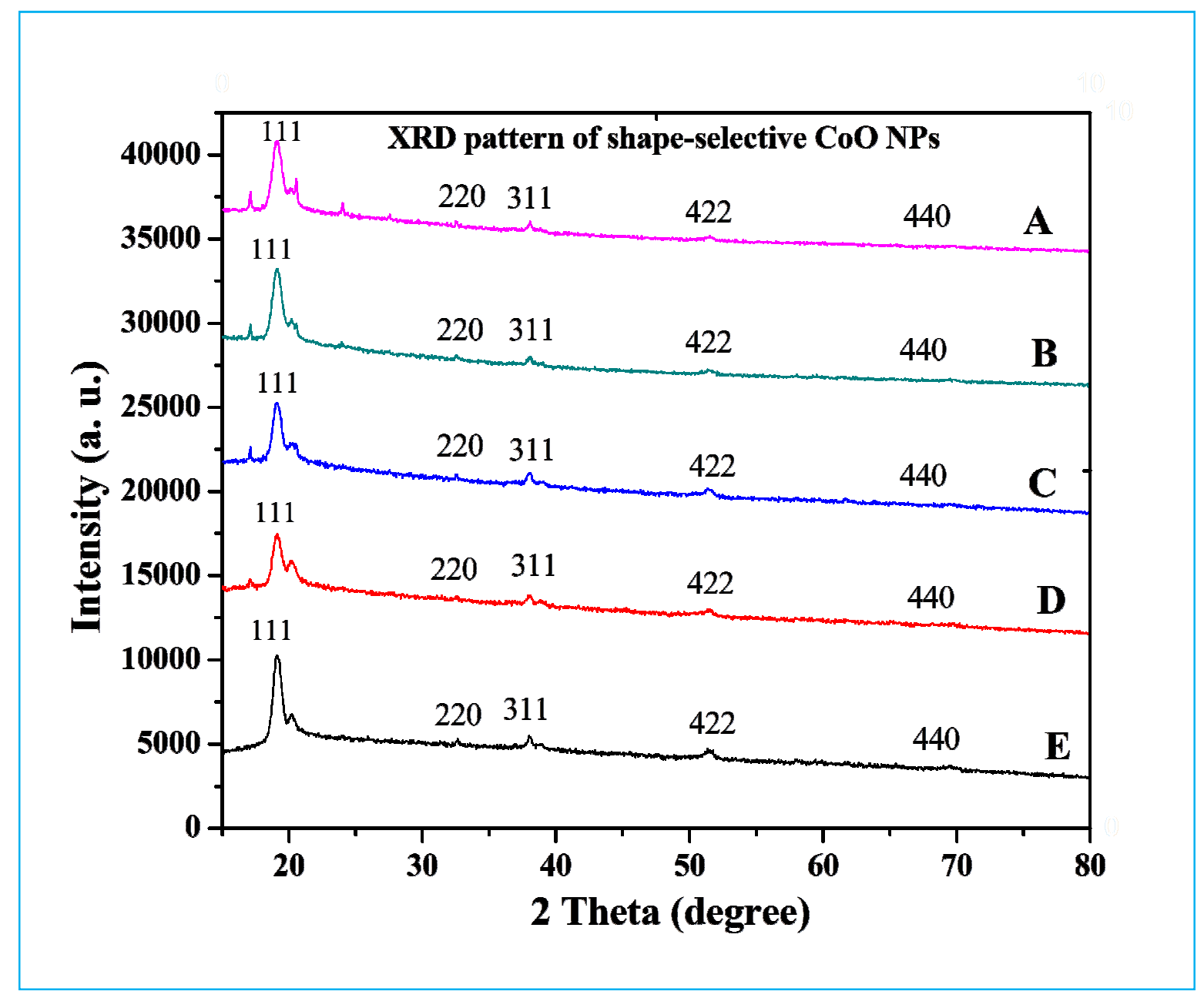

Figure 5 

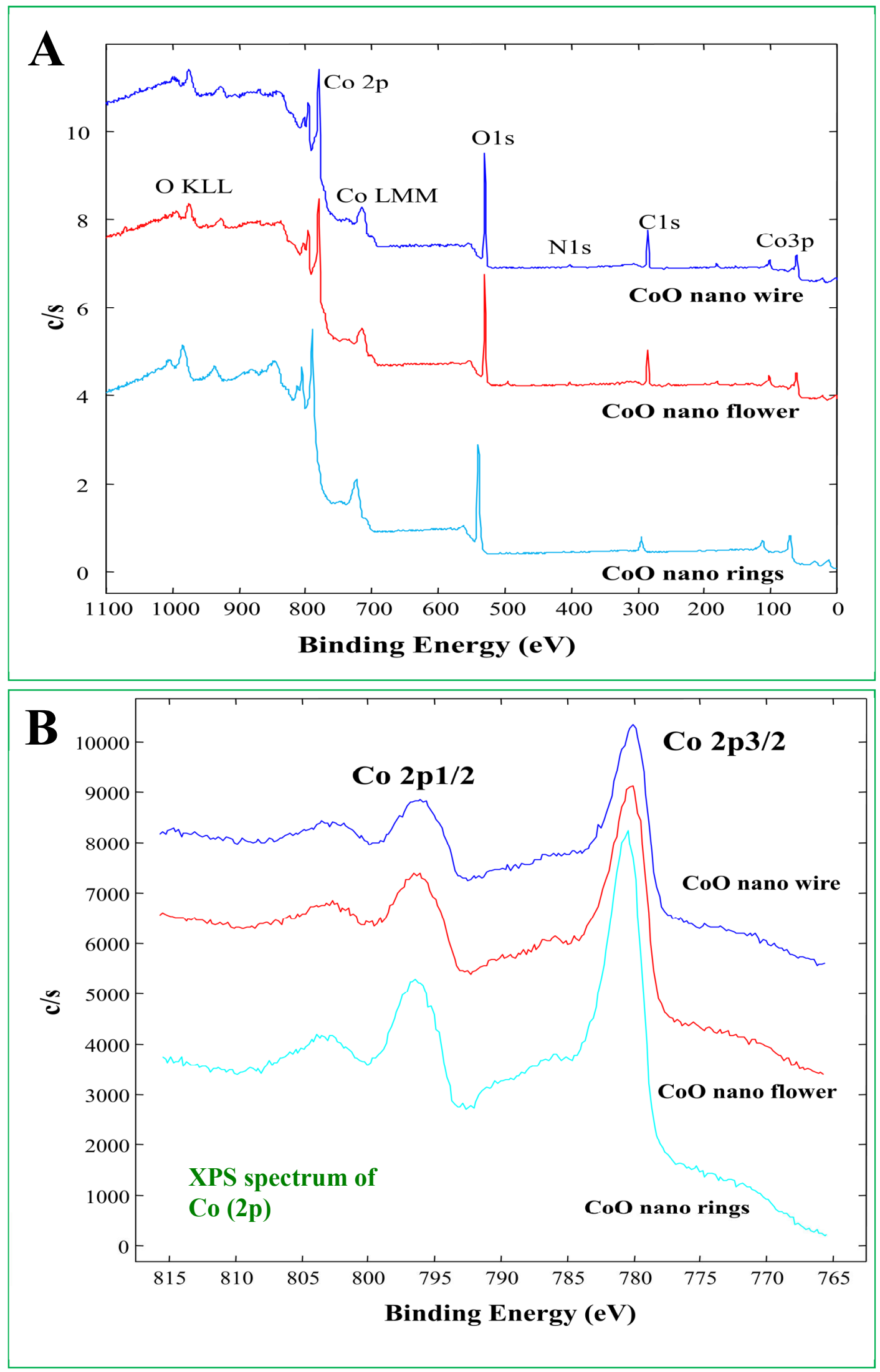

Figure 6, A-B 


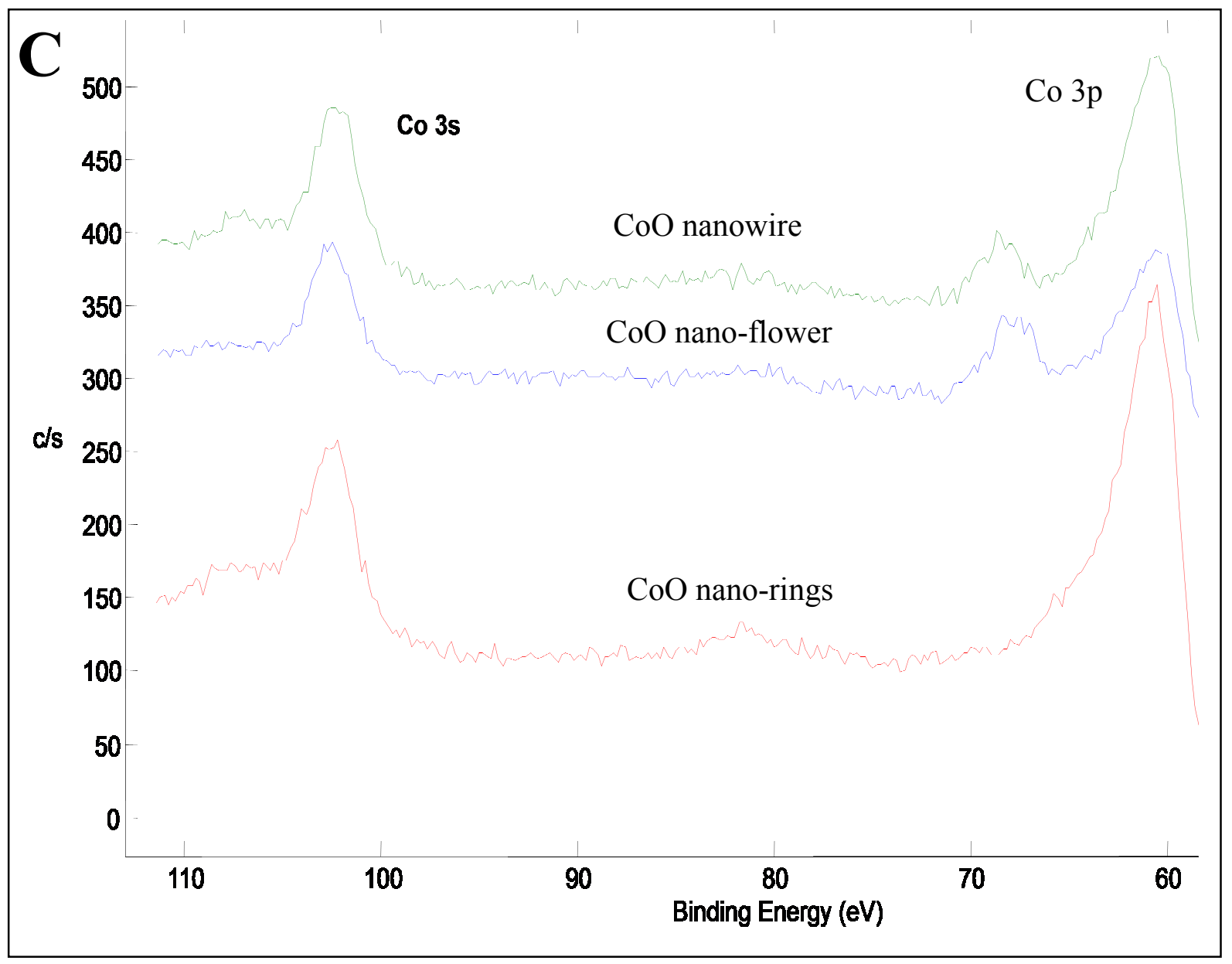

Figure 6, C 

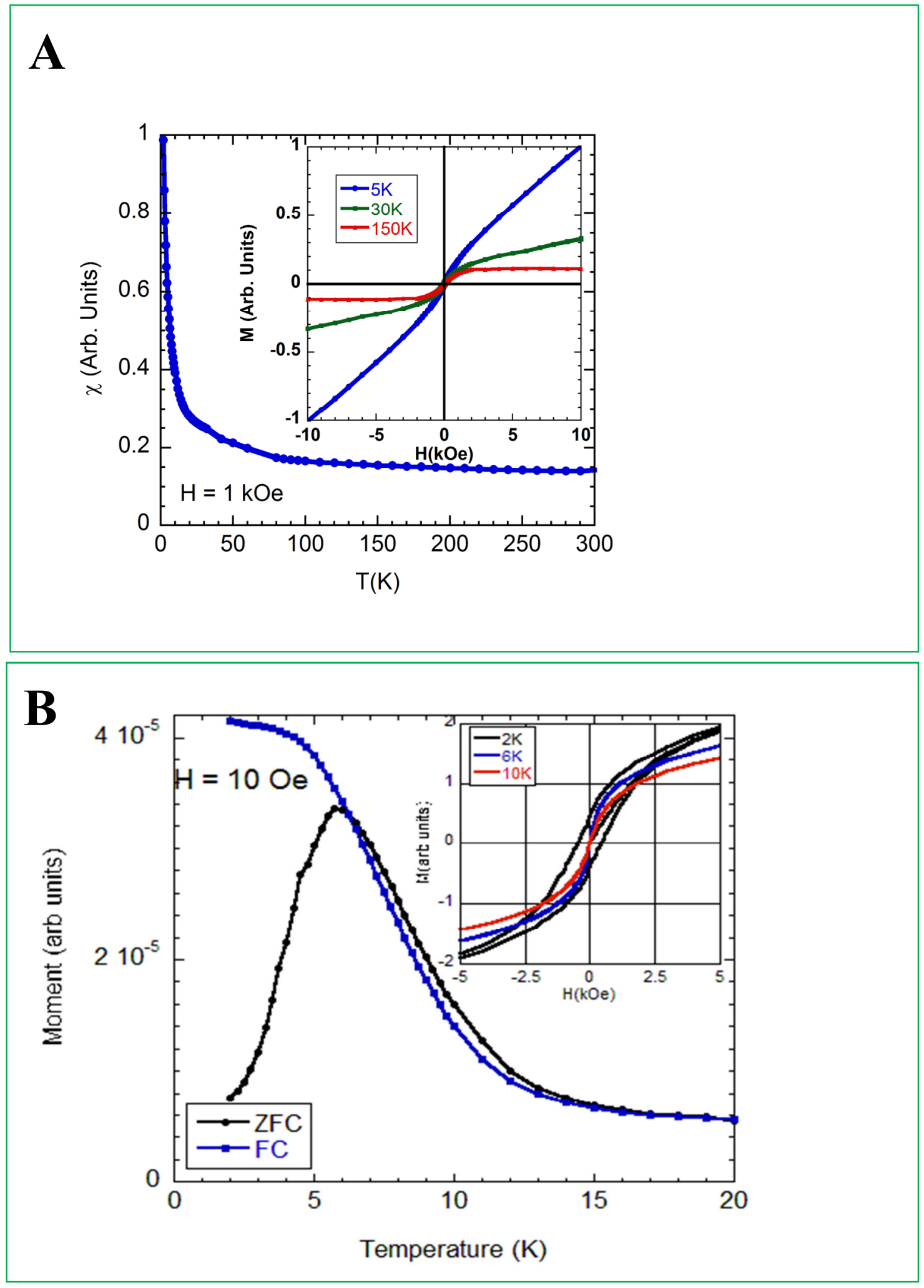

Figure 7, A-B 


\begin{tabular}{|c|c|c|c|c|c|c|c|c|}
\hline Set No & $\begin{array}{l}\text { Final Conc. } \\
\text { of CTAB (M) }\end{array}$ & $\begin{array}{l}\text { Final conc. of } \\
\text { Co(II) ions } \\
\text { (M) }\end{array}$ & $\begin{array}{l}\text { Final conc. of } \\
\text { 2,7-DHN (M) }\end{array}$ & $\begin{array}{c}\text { Final conc. of } \\
\mathrm{NaOH}(\mathrm{M})\end{array}$ & $\begin{array}{c}\text { Time of } \\
\text { UV } \\
\text { photo- } \\
\text { irradiati } \\
\text { on (h) } \\
\end{array}$ & $\begin{array}{c}\text { Shape of } \\
\text { the CoO } \\
\text { NPs }\end{array}$ & $\begin{array}{l}\text { Size of the } \\
\text { CoO NPs }\end{array}$ & $\begin{array}{c}\text { Shape } \\
\text { distribution }\end{array}$ \\
\hline 1 & $7.51 \times 10^{-4}$ & $1.05 \times 10^{-3}$ & $1.35 \times 10^{-3}$ & $7.51 \times 10^{-3}$ & 4 & Sphere & $\begin{array}{c}\sim 80 \pm 10 \\
\mathrm{~nm}\end{array}$ & $\begin{array}{c}100 \% \\
\text { spheres }\end{array}$ \\
\hline 2 & $7.75 \times 10^{-3}$ & $9.3 \times 10^{-4}$ & $1.24 \times 10^{-3}$ & $7.75 \times 10^{-3}$ & 4 & $\begin{array}{l}\text { Ring } \\
\text { like }\end{array}$ & $\begin{array}{c}\sim 25 \pm 5 \\
\mathbf{n m}\end{array}$ & $\begin{array}{c}100 \% \\
\text { ring like }\end{array}$ \\
\hline 3 & $7.40 \times 10^{-3}$ & $1.03 \times 10^{-3}$ & $1.48 \times 10^{-3}$ & $7.40 \times 10^{-3}$ & 4 & $\begin{array}{l}\text { Flower } \\
\text { like }\end{array}$ & $\begin{array}{c}\sim 350 \pm \\
50 \mathrm{~nm}\end{array}$ & $\begin{array}{c}95 \% \\
\text { flower } \\
\text { like } \\
\end{array}$ \\
\hline 4 & $7.70 \times 10^{-2}$ & $1.05 \times 10^{-3}$ & $1.48 \times 10^{-3}$ & $7.40 \times 10^{-3}$ & 4 & $\begin{array}{c}\text { Wire } \\
\text { (short) }\end{array}$ & $\begin{array}{c}\text { length } \\
\sim 210 \pm 10 \\
\text { nm and } \\
\text { diameter } \\
\sim 45 \pm 5 \\
\text { nm. }\end{array}$ & $\begin{array}{l}100 \% \\
\text { wires }\end{array}$ \\
\hline 5 & $7.40 \times 10^{-2}$ & $8.46 \times 10^{-4}$ & $1.38 \times 10^{-3}$ & $7.69 \times 10^{-3}$ & 4 & $\begin{array}{l}\text { Wire } \\
\text { (long) }\end{array}$ & $\begin{array}{c}\text { length } \\
\sim \mathbf{3 0 0} \pm \mathbf{1 0} \\
\text { nm and } \\
\text { diameter } \\
\sim \mathbf{3 5} \pm \mathbf{3} \\
\mathbf{n m} .\end{array}$ & $\begin{array}{l}100 \% \\
\text { wires }\end{array}$ \\
\hline
\end{tabular}

Table 1 


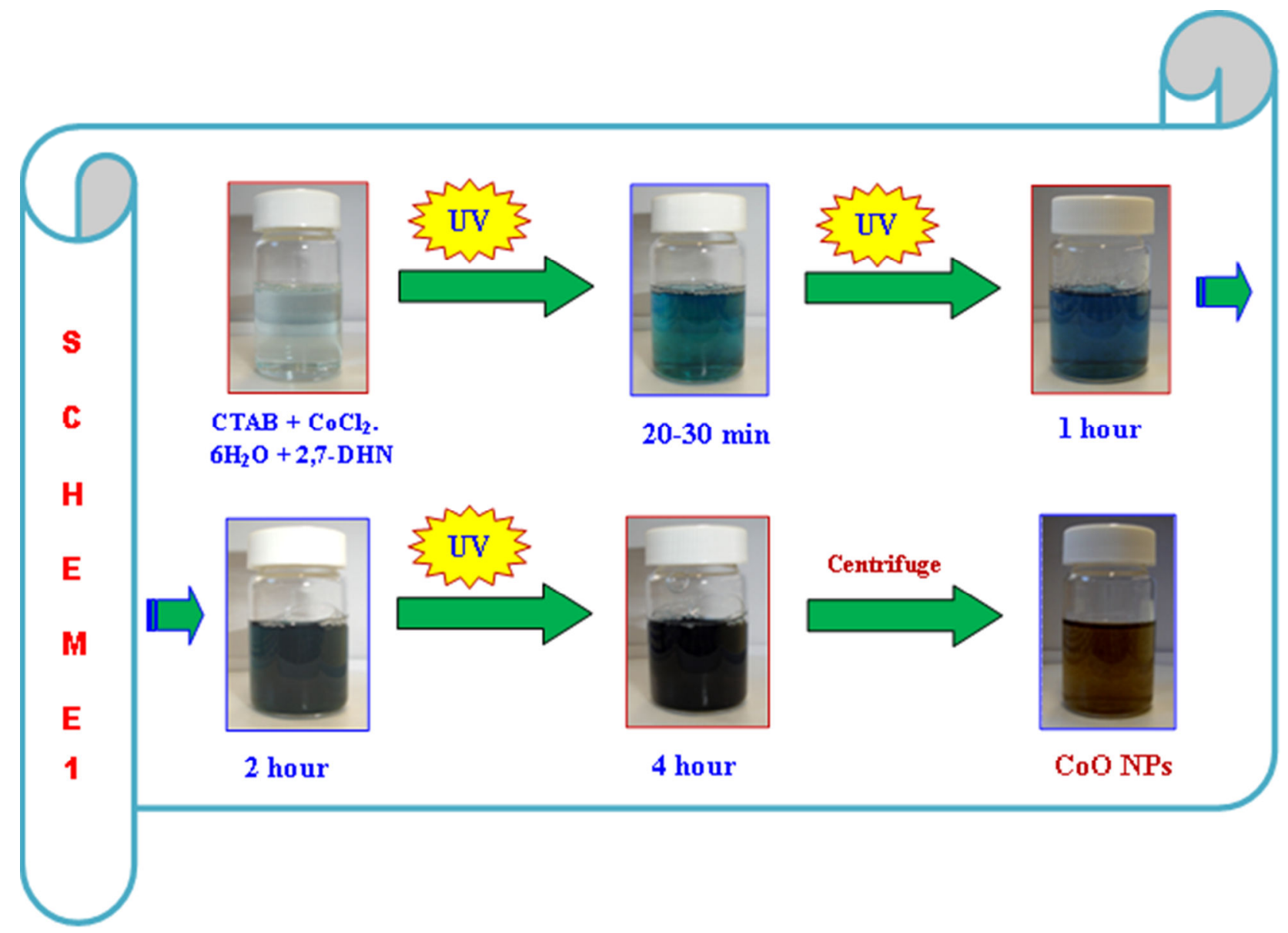

Scheme 1 


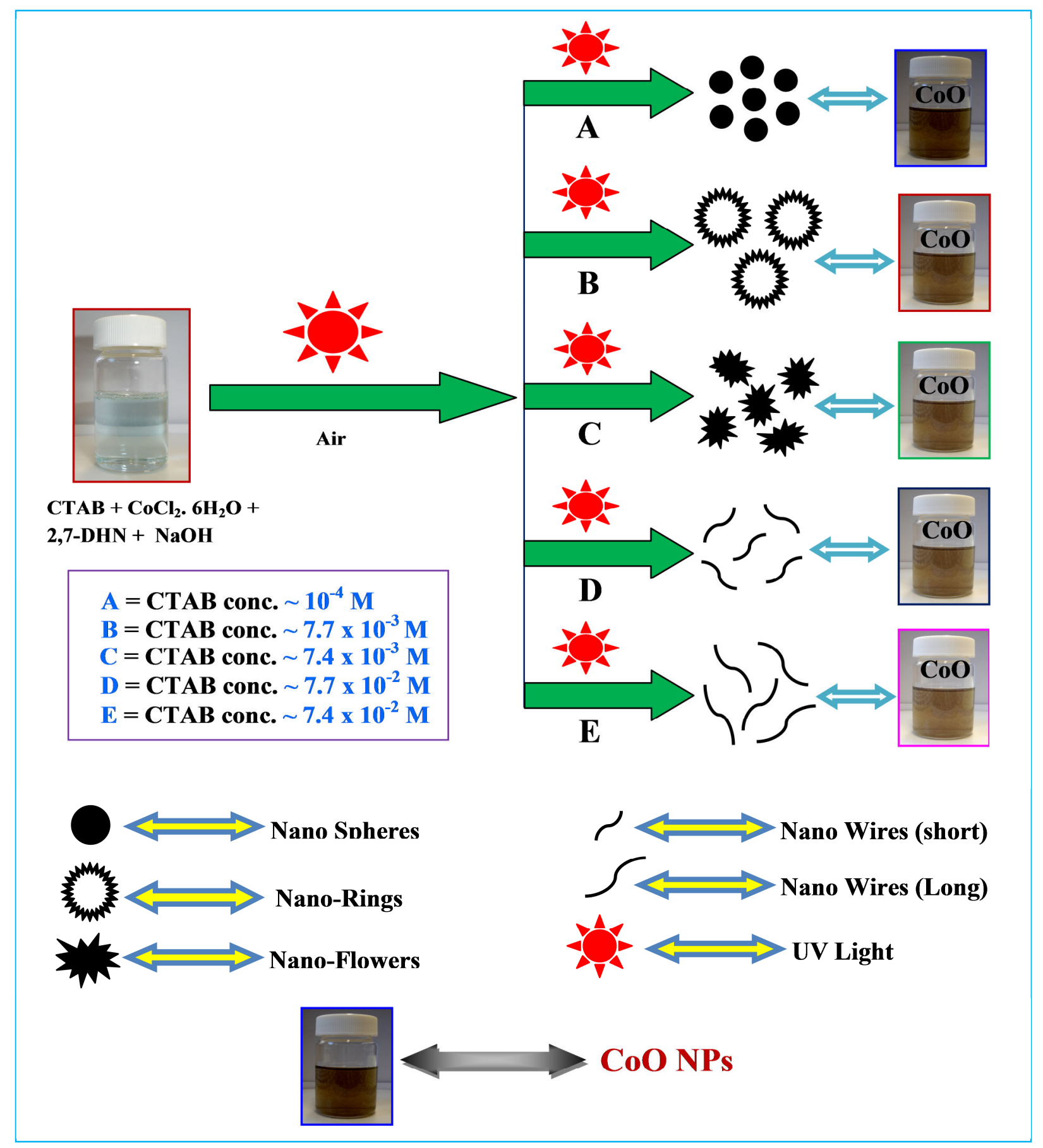

Scheme 2 


\title{
Graphical Abstract
}

\section{Shape-influenced Magnetic Properties of CoO Nanoparticles}

\author{
Subrata Kundu, ${ }^{\dagger+*}$ Art J. Nelson, ${ }^{\#}$ Scott McCall, \\ Anthony van Buuren, ${ }^{\#}$ and Hong Liang :* $^{*}$
}

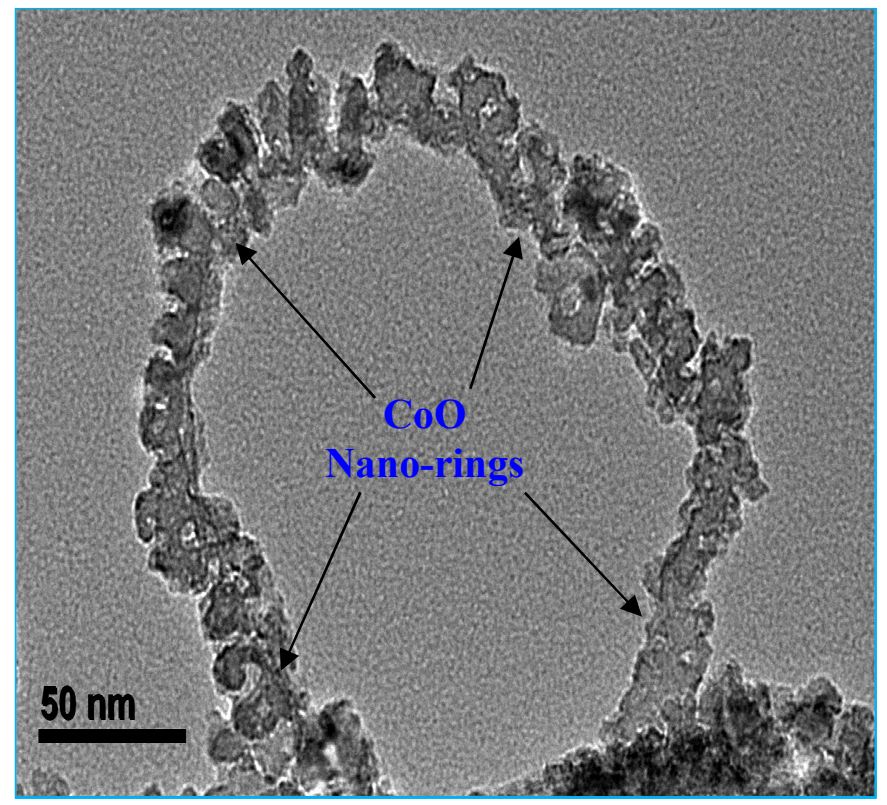

Using a wet chemical approach, $\mathrm{CoO}$ nanospheres, nano-rings, nano-flowers, and nanowires of different sizes were generated. Among those, nano-rings show ferromagnetic behavior below $6 \mathrm{~K}$ while the nanospheres remain paramagnetic down. X-ray photoelectron spectroscopy for Co $2 p$, $3 p$ and $3 \mathrm{~s}$ core-levels indicates the paramagnetic high-spin Co(II) electronic configuration. This finding reveals the optical, electronic, and magnetic behavior of $\mathrm{CoO}$ nanoparticles (NPs) that opens opportunities for future applications as catalysts precursors for making pigments, lithiumion battery materials, or as solid state sensors as anisotropy source for magnetic recording. 


\title{
Supporting Information for
}

\section{Shape-influenced Magnetic Properties of CoO Nanoparticles}

\author{
SubrataKundu, ${ }^{+* *}$ Art J. Nelson, ${ }^{\#}$ Scott McCall, ${ }^{\#}$ \\ Anthony van Buuren, ${ }^{\#}$ and Hong Liang ${ }^{* *}$
}

\begin{abstract}
*Materials Science \& Mechanical Engineering, Texas A\&M University, College Station, TX 77843-3123 (USA)

\#Lawrence Livermore National Laboratory, Physical \& Life Sciences Directorate, Condensed Matter \& Materials Division, 7000 East Avenue, Livermore, CA 94550 (USA).

${ }^{\dagger}$ Electrochemical Materials Science (ECMS) Division, CSIR-Central Electrochemical Research Institute (CSIR-CECRI), Karaikudi-630006, Tamil Nadu, INDIA.
\end{abstract}

*CorrespondingE-mails: skundu@cecri.res.in and hliang@tamu.edu,Phone-979-862-2623, Fax - 979-845-3081

\section{Synthesis Details:}

\section{Chemical and Instruments}

Cetyltrimethylammonium bromide (CTAB, 99\%) was purchased from Sigma-Aldrich and used as received. The 2,7-dihydroxynapthalene (2,7-DHN) was purchased from SigmaAldrich and was re-crystallized in hot water. The hydratedcobalt (II) chloride, hexahydrate $(\mathrm{CoCl} 2 \cdot 6 \mathrm{H} 2 \mathrm{O})$ and sodium hydroxide $(\mathrm{NaOH})$ were obtained from Sigma-Aldrich and used asreceived. De-ionized (DI) water was used for the entire synthesis.

The UV-visible (UV-vis) absorption spectra were recorded in a Hitachi (model U-4100) UV-vis-NIR spectrophotometer equipped with a $1 \mathrm{~cm}$ quartz cuvette holder for liquid samples. A high resolution-transmission electron microscope (HR-TEM) (ZEOL ZEM 2010) was used at an 
accelerating voltage of $200 \mathrm{kV}$. The energy dispersive X-ray spectrum (EDS) was recorded with an Oxford Instruments, INCA energy system connected with the TEM. The XRD analysis was done with a scanning rate of $0.020 \mathrm{~s}-1$ in the $2 \theta$ range $15-80^{\circ}$ using a Bruker-AXS D8 Advanced Bragg-Brentano X-ray Powder Diffractometer with $\mathrm{Cu} K \alpha$ radiation $(\lambda=0.154178 \mathrm{~nm})$. The $\mathrm{X}$ ray photoelectron spectroscopy (XPS) analysis was carried out using a focused monochromatic Al Ka X-ray $(1486.7 \mathrm{eV})$ source for excitation and a spherical section analyzer. A $100 \mu \mathrm{m}$ diameter X-ray beam was used for analysis. The X-ray beam is incident normal to the sample and the X-ray detector is at $45^{\circ}$ away from the normal. The analysis area on the sample is 0.4 by $0.7 \mathrm{~mm}$. The pass energy was $23.5 \mathrm{eV}$ giving an energy resolution of $0.3 \mathrm{eV}$ that when combined with the $0.85 \mathrm{eV}$ full width at half maximum (FWHM) $\mathrm{Al} \mathrm{K \alpha}$ line width gives a resolvable XPS peak width of $1.2 \mathrm{eV}$ FWHM. Deconvolution of non-resolved peaks was accomplished using Multipack 9.2 (PHI) curve fitting routines. Binding energies were referenced to the $\mathrm{C} 1 \mathrm{~s}$ photoelectronline arising from adventitious carbon at $284.8 \mathrm{eV}$. Low energy electrons were used for specimen neutralization.Magnetic measurements were made in a commercial SQUID magnetometer where a reasonable amounts of NPs in solution were deposited onto a small piece of a chemwipe, dried, and placed in a polypropylene sample holder. The background contribution from the sample holder, including the chemwipe was constant, weakly diamagnetic, and accounted for less than $0.1 \%$ of the measured response in low magnetic fields. Samples were initially zero field-cooled (ZFC) and measured from a base temperature of $2 \mathrm{~K}$ in a magnetic field of $10 \mathrm{Oe}$, followed by a field-cooled (FC) measurement in 10 Oe. Isothermal magnetization measurements were performed and the hysteresis was observed only below 5K.A xenon lamp from Newport Corporation at a wavelength of $270 \mathrm{~nm}$ on the sample was used for UV photo-irradiation. The approximate intensity was $18 \mu \mathrm{W}$ and the bandwidth of irradiation was $270 \pm 10 \mathrm{~nm}$. The distance of the sample from the light source was $\sim 16.6 \mathrm{~cm}$. The sample was placed over a wooden box with a stand to make the light fall directly onto it.

\section{Synthesis of Shape-selective CoO NPs}

Shape-selective CoO NPs were synthesized by altering the concentration of CTAB and $\mathrm{Co}(\mathrm{II})$ ions in the reaction mixture containing $\mathrm{CoCl} 2 \cdot 6 \mathrm{H} 2 \mathrm{O}, 2,7-\mathrm{DHN}, \mathrm{CTAB}$, and $\mathrm{NaOH}$. For a typical synthesis process, $50 \mathrm{~mL}$ of CTAB $(0.1 \mathrm{M})$ was mixed with $7 \mathrm{~mL}$ of $10-2 \mathrm{M} \mathrm{CoCl} 2$. 
$6 \mathrm{H} 2 \mathrm{O}$ solution. Then $10 \mathrm{~mL}$ of $10-2 \mathrm{M} 2,7-\mathrm{DHN}$ solution and $500 \mu \mathrm{L}$ of $\mathrm{NaOH}(1 \mathrm{M})$ solution were added. The solution mixture stirred for $30 \mathrm{sec}$ using magnetic stirrer. Then the mixture was placed in front of UV light source and UV-irradiated continuously for 4 hours with constant stirring. The process produces the $\mathrm{CoO}$ nanorods. For the synthesis of other shapes we varied the concentrations of CTAB and $\mathrm{Co}$ (II) ions keeping the UV photo-irradiation time fixed. The final concentration of all the chemicals and other reaction parameters are given in Table 1. After the addition of $\mathrm{NaOH}$ and stirring for $30 \mathrm{sec}$, the solution is light bluish. During UV-irradiation for 20-30 min, the solution is bluish green color, after 2 hours it became deep blue, after 3 hours it became bluish black and finally after 4 hours it is blackish in color. The solution mixture was centrifuged at $6000 \mathrm{rpm}$ for $30 \mathrm{~min}$ and again at $4000 \mathrm{rpm}$ for $15 \mathrm{~min}$ to remove excess $\mathrm{CTAB}$ and other chemicals from the NPs solution. The precipitated light brown CoO NPs was redispersed in DI water and stored in dark place at $4{ }^{\circ} \mathrm{C}$ in a refrigerator and found to be stable for more than a month without changing any optical properties.

\section{Characterization of Shape-selective CoO NPs}

The CTAB stabilized CoO NPs were characterized using TEM, EDS, XRD, XPS, and magnetization measurements. The sample for TEM analysis was prepared by placing a drop of the corresponding $\mathrm{CoO}$ NPs solution onto a carbon-coated $\mathrm{Cu}$ TEM grid followed by slow evaporation of the solvent at ambient condition. The EDS analysis was done from the samesamples during the TEM measurement. The samples for the XRD and XPS analyses were prepared on a glass substrate for making thin films. Before the NP deposition, the wafers were cleaned thoroughly in acetone and sonicated for $30 \mathrm{~min}$. Then the substrate was dried and the clean substrate used for $\mathrm{CoO}$ NPs deposition. After deposition, the sample was dried in a vacuum chamber. Final samples were prepared with 6-8 depositions, dried, and then analyzed using XRD and XPS techniques. The samples for magnetic measurements were made by depositing a reasonable amounts of NPs solution onto a small piece of a chemwipe, dried, and placed in a polypropylene sample holder. 